\title{
Heavy Metal Lead Removal by Biosorption - A Review
}

\author{
Chris Sheba M. \& Monica Nandini G. K. \\ Department of Civil Engineering, \\ Sri Ramakrishna Institute of Technology, \\ Pachapalayam, Perur Chettipalayam, \\ Coimbatore - 641 010, India.
}

\begin{abstract}
Biosorption technique is increasingly researched and used in the biosorption of various heavy metals from industrial effluents over the past decades. Factors such as temperature, $\mathrm{pH}$, initial metal ion concentration, biomass concentration and the metal affinity to the biosorbent, affect the efficiency of the biosorption capacity. Biosorbents such as large scale industrial fermentation by-products, agricultural waste, seaweeds, etc are used for lead removal. This article reviews the various research on biosorbents reported from 2013 - 2016, for the biosorption capacity \& the kinetics and equilibrium isotherm models that the sorbents follow for optimum process. The thermodynamics of the process of certain biosorbents have also been selectively overviewed.
\end{abstract}

Keywords - Lead; Biosorption; Biosorbent; Isotherm; Adsorption capaciy.

\section{INTRODUCTION}

Heavy metal pollution is one of the major environmental problems prevalent today. Lead exists as $\mathrm{Pb}$ (II) and is toxic to living organisms in its concentrated forms. It is a metabolic poison which accumulates in the blood [1], mineralizing tissues (bones and teeth) [2] and soft tissues of human body (liver, kidneys, lungs, brain, spleen, muscles, and heart) [3]. It is an enzyme inhibitor which forms complexes with Oxogroups in enzymes to affect virtually all steps in the synthesis of haemoglobin and porphyria metabolism [4]. It causes various health problems such as anaemia, Alzheimer's disease, nervous system deterioration, failure of kidneys, bone problems and nervous system deterioration [5]. It affects infants and small children as well $[6,7]$. The World Health Organization considers lead to be in the top ten chemical of public concern. [8] The permissible limit of lead in drinking water and surface water intended for drinking, as set by EU, US-EPA and WHO are $0.010,0.015$ and $0.010 \mathrm{mgL}^{-1}$ respectively [9, 10 and 11$]$.

Industrial effluents containing high concentrations of lead are introduced in the water bodies from mining, manufacturing of batteries, paint, pigments, burning of coal, etc. Thus the removal and recovery of heavy metals from effluent streams are essential to the protection of the environment.

\section{BIOSORPTION PROCESS}

Biosorption is a property of certain types of inactive, dead, microbial biomass to bind and concentrate heavy metals from even dilute aqueous solutions. It is a metabolically passive process, meaning it does not require energy, and the amount of contaminants that a sorbent can remove is dependent on kinetic equilibrium and the composition of the sorbents cellular surface [12].
The biosorption process involves a solid phase (sorbent or biosorbents; biological material) and a liquid phase (solvent, normally water) containing the dissolved metal ions (adsorbate, heavy metal) to be sorbent. Due to the higher affinity of the adsorbent for the adsorbate species, the latter is attracted and bound by different mechanisms such as ion exchange, complexation, chelation, microprecipitation, etc. The process continues till equilibrium is established between the amount of solid-bound adsorbate species and its portion remaining in the solution. The degree of adsorbent affinity for the adsorbate determines its distribution between the solid and liquid phases [13].

\section{A. Advantages of Biosorption Process}

Compared with the conventional heavy metal removal methods, the potential advantages of biosorption process includes [14]: • High selectivity for recovery and removal of specific heavy metals • High affinity thereby removing residual metals to $<1 \mathrm{ppb} \bullet$ Ability to treat large volumes of mixed wastes and wastewater with multiple heavy metals • Use of naturally renewable biomaterial that are cheap and abundant, reducing the need for expensive reagents $\bullet$ Wide range of physicochemical operational conditions including $\mathrm{pH}$, temperature and also presence of other ions • Relatively low investment and operational cost but with enhanced recovery of bound heavy metals ions • The hazardous waste produces is greatly reduced in volume.

\section{B. Factors Affecting Biosorption of Metals}

Biosorption depends on many factors that are related to the biomass and metal; while some other factors are related to environmental conditions.

1) Temperature: For most biomass, the biosorption activity occurs at the room temperature. However in certain cases, the temperature plays a major role in determining the optimum conditions for the process. In the biosorption study using peanut shells, its capacity to adsorb $\mathrm{Pb}(\mathrm{II})$ decreased as the temperature increased. It was also observed that the process was exothermic and spontaneous [15].

2) $\mathrm{pH}: \mathrm{pH}$ dependent experiments were advised not to be conducted at $\mathrm{pH}>5$ in order to avoid precipitation of $\mathrm{Pb}$ ions as hydroxides [16]. Since protons can be adsorbed or released in the biosorption process, the $\mathrm{pH}$ affects the metal ion solubility and the total charge of the biosorbent [17]. This behavior also depends on the functional groups present on the biomass' cell wall. 
3) Initial metal ion and biosorbent concentrations: The concentration of exchangeable sites on the cell wall of the biosorbents affects its removal capacity. At minute initial concentrations of metal ions, $1 \mathrm{~g}$ (say) of biomass would be enough for complete removal by biosorption. But when the metal ion concentration increases, the exchangeable sites in the $1 \mathrm{~g}$ of biomass will be not be sufficient for biosorption, resulting in obvious reduced removal capacity. The study by Hasan et al, 2016 [18], the gradual increase in equilibrium uptake of the biosorbent with the increase in initial metal ion concentration was reported. This was probably due to the higher interaction between the exchangeable sites of the biosorbent and the metal ions. Hence the metal ion to adsorbent ratio plays a critical role in the biosorption process. In contrast, the presence of $\mathrm{NaCl}$ reduced the sorption efficiency due to the competition for binding sites between the $\mathrm{Na}^{+}$and divalent cations [19]. Hence the biosorption capacity could be determined by the temperature, $\mathrm{pH}$, initial metal ion concentration, biomass concentration and the metal affinity to the biosorbent.

\section{BIOSORBENTS}

The first major challenge for the biosorption field was to choose the most promising types of biomass/ biosorbent from enormously available and inexpensive biomaterials. Even though several materials of biological origin bind heavy metals, biomaterials with sufficiently high metal-binding capacity and selectivity for heavy metals are appropriate for full-scale biosorption process [20].

\section{A. Types of Biomass}

The types of biomass used for biosorption process includes large scale industrial by-products of fermentation (e.g. mold Rhizopus sp. [21, 22] and bacterium Bacillus sp. [23]), agricultural waste (like husks of rice [24], coffee [25], etc), seaweeds (e.g. algae) [26], etc. These biomass types can accumulate in excess $25 \%$ of their dry weight in heavy metals. Moreover chemically modified biomass shows improved biosorption capacity. The functionalized Saccharomyces cerevisiae cell with biogenic intracellular $\mathrm{CaCO}_{3}$ mineral scaffold was efficient for removing $\mathrm{Pb}$ (II) ions from aqueous solutions at the maximum removal capacity of $116.69 \mathrm{mg}$ $\mathrm{g}^{-1}[27] . \mathrm{HNO}_{3}$ modified Phytolacca americana was found to have higher uptake capacity than the original $P$. americana [28]. The different lead removal capacities of several biosorbents under optimum conditions are summarized in table I.

For the biomass to be effective biosorbents, the vital evaluation elements for an adsorption process are the mechanism of biosorption and the reaction rate. Biosorbate uptake rate determines the time required for completing the adsorption reaction and can be described from the kinetic analysis.

\section{KINETICS OF ADSORPTION}

To explain the solid/liquid biosorption processes, Lagergren first described the process and presented the first order rate equation. In order to differentiate the kinetic equations based on adsorption capacity from solution concentration, the Lagergren's first order rate equation is also known as the pseudo - first order kinetic equation [29, 30].The pseudo - second order kinetic equation [31] and intraparticle diffusion model is also used to predict the sorption kinetics. The pseudo - first order and pseudo second order define the metal interactions (physisorption or chemisorptions). The intraparticle diffusion model defines the internal diffusion that establishes the adsorption rate in most of the liquid systems [32]. The linear equations of these kinetic models are presented in table II

Lagergren's pseudo first order model describes the rate of adsorption reaction to be dependent on the available concentration of adsorbent. In previous studies, this model was not able to correlate with the experimental values, probably due to the time lag causing the presence of boundary layer that controls at the beginning of the biosorption process [37]. Pseudo second order model indicates chemisorptions to be the rate limiting step [42]. Biosorption of lead using corn husk [37], peanut shells [15], C. interruptus [38], Marula seed husk (S. birrea) [39], P. americana [28], maize stover [40], nanostructured cedar leaf ash [35], cashew nut shell (A. occidentale L.) [41], Casuarina Leaf Powder [16], $S$. melongena leaf powder [42], and Castor leaf ( $R$. communis) [43] have been reported to follow pseudo second order model for adsorption.

TABLE I. L LEAD REMOVAL CAPACITIES OF VARIOUS

\begin{tabular}{|c|c|c|c|c|}
\hline \multicolumn{5}{|c|}{ TS } \\
\hline \multirow[b]{2}{*}{ Biosorbent } & \multicolumn{2}{|c|}{ Operation Conditions } & \multirow{2}{*}{$\begin{array}{l}\text { Biosorption } \\
\text { Capacity, q }\end{array}$} & \multirow[b]{2}{*}{ Ref } \\
\hline & $\mathrm{pH}$ & $\begin{array}{c}\text { Biomass } \\
\text { Concentration }\end{array}$ & & \\
\hline Peanut shells & 5.5 & $2 \mathrm{~g} / \mathrm{L}$ & $33 \mathrm{mg} / \mathrm{g}$ & [15] \\
\hline Coffee Husk & 5 & $2 \mathrm{~g}$ & $50 \mathrm{mg} / \mathrm{g}$ & [25] \\
\hline P. americana $\mathrm{L}$. & \multirow[b]{2}{*}{6} & \multirow[b]{2}{*}{$20 \mathrm{~g} / \mathrm{L}$} & $10.83 \mathrm{mg} / \mathrm{g}$ & \multirow[b]{2}{*}{ [28] } \\
\hline $\begin{array}{l}\mathrm{HNO}_{3} \text { modified } P . \\
\text { americana } \mathrm{L} \text {. }\end{array}$ & & & $12.66 \mathrm{mg} / \mathrm{g}$ & \\
\hline $\begin{array}{c}\text { Marula seed husk } \\
\text { (Sclerocarya birrea) }\end{array}$ & 5 & $0.8 \mathrm{~g}$ & $20 \mathrm{mg} / \mathrm{g}$ & [39] \\
\hline Castor Leaf Powder & 5 & $0.02 \mathrm{~g}$ & $0.327 \mathrm{mmol} / \mathrm{g}$ & [44] \\
\hline Ulva lactuca & 5 & $0.8 \mathrm{~g} / \mathrm{L}$ & $68.9 \mathrm{mg} / \mathrm{g}$ & [50] \\
\hline Sargassum ilicifolium & 3.7 & $0.2 \mathrm{~g} / \mathrm{L}$ & $195 \pm 3.3 \mathrm{mg} / \mathrm{g}$ & [51] \\
\hline $\begin{array}{c}\text { (live) Spirulina } \\
\text { (Arthospira) maxima, }\end{array}$ & \multirow{3}{*}{7} & \multirow{3}{*}{$0.24(\mathrm{~g} / \mathrm{L})$} & $27.25 \mathrm{mg} / \mathrm{gdw}$ & \multirow{3}{*}{52} \\
\hline $\begin{array}{c}\text { (live) Spirulina } \\
\text { (Arthospira) indica, }\end{array}$ & & & $28.2 \mathrm{mg} / \mathrm{gdw}$ & \\
\hline $\begin{array}{c}\text { (live) Spirulina } \\
\text { (Arthospira) platensis }\end{array}$ & & & $28.25 \mathrm{mg} / \mathrm{gdw}$ & \\
\hline $\begin{array}{c}\text { Curtobacterium sp. } \\
\text { FM01 }\end{array}$ & 6 & $1 \mathrm{mg}$ & $180.6 \mathrm{mg} / \mathrm{g}$ & [53] \\
\hline $\begin{array}{c}\text { Streptomyces fradiae } \\
\text { biomass }\end{array}$ & 5 & $1 \mathrm{~g} \mathrm{dm}^{-3}$ & $138.88 \mathrm{mg} / \mathrm{g}$ & [54] \\
\hline $\begin{array}{c}\text { Sophora japonica } \\
\text { pods }\end{array}$ & $\begin{array}{c}6.0- \\
7.0\end{array}$ & $0.5 \mathrm{~g} / \mathrm{L}$ & $25.13 \mathrm{mg} / \mathrm{g}$ & [55] \\
\hline Otostegia persica & 5.5 & $1.06 \mathrm{~g} / \mathrm{L}$ & $17.3 \mathrm{mg} / \mathrm{g}$ & [56] \\
\hline
\end{tabular}

TABLE II. KINETIC MODEL LINEAR EQUATION [33]

\begin{tabular}{|c|cc|c|}
\hline Kinetic Model & Linear Equation & $\begin{array}{c}\text { Eqn. } \\
\text { No. }\end{array}$ & $\begin{array}{c}\text { Plot } \\
\text { representation }\end{array}$ \\
\hline $\begin{array}{c}\text { Pseudo - first } \\
\text { order }\end{array}$ & $\begin{array}{c}\log \left(q_{e}-q_{t}\right) \\
=\log \left(q_{e}\right)-\frac{k_{1}}{2.303} t\end{array}$ & $\begin{array}{c}\log \left(q_{e}-q_{t}\right) \\
\text { vs } t\end{array}$ \\
\hline $\begin{array}{c}\text { Pseudo - second } \\
\text { order }\end{array}$ & $\frac{t}{q_{t}}=\frac{k_{2}}{k_{2} q_{e}{ }^{2}}+\frac{t}{q_{e}}$ & $(2)$ & $\frac{t}{q_{t}}$ vs $t$ \\
\hline $\begin{array}{c}\text { Intra - particle } \\
\text { diffusion }\end{array}$ & $q_{t}=K_{i d} t^{0.5}+C$ & $(3)$ & $q_{t}$ vs $t^{0.5}$ \\
\hline
\end{tabular}


Where the parameters are $\mathrm{q}_{\mathrm{e}}(\mathrm{mg} / \mathrm{g})$ and $\mathrm{q}_{\mathrm{t}}(\mathrm{mg} / \mathrm{g})$ are the adsorption capacity at equilibrium and time $\mathrm{t}$ (min), respectively; $\mathrm{k}_{1}(1 / \mathrm{min})$ and $\mathrm{k}_{2}(\mathrm{~g} /(\mathrm{mg} \min ))$ are the pseudofirst and pseudo-second order rate constants, respectively; $\mathrm{K}_{\mathrm{id}}$ $\left(\mathrm{mg} / \mathrm{g} \mathrm{min}^{0.5}\right)$ is the intraparticle diffusion rate constant and $\mathrm{C}$ is the intercept.

The overall rate of adsorption can be described by the following three steps: (1) fluid transport, (2) film diffusion, (3) surface diffusion. If the nature of diffusion could not be identified by the first and second pseudo order model the intra-particle diffusion model is investigated. If the intraparticle diffusion is the sole rate-limiting step, intercept of the line, in the plot representation, should pass through the origin, i.e. $\mathrm{C}=0$ [34]. The study on rapeseed [33] suggests that it underwent pseudo second order reaction and intra-particle diffusion.

\section{EQUILIBRIUM MODELS OF ADSORPTION}

In order to understand the relationship between the concentrations of lead adsorbed to its equilibrium concentration in the biosorbent solution, kinetic isotherms are applied. Isotherms define the adsorption efficiency. The different isotherm models along with its linear equation are given in table III.

TABLE III. BIOSORPTION ISOTHERM MODEL - LINEAR EQUATION [33]

\begin{tabular}{|c|c|c|c|}
\hline Isotherm Model & Linear Equation & $\begin{array}{l}\text { Eqn. } \\
\text { No. }\end{array}$ & Plot representation \\
\hline Freundlich [33] & $\begin{array}{l}\log q_{e} \\
=\log K_{F} \\
+{ }_{n}^{1} \log C_{e}\end{array}$ & (4) & $\log q_{e} v s \log C_{e}$ \\
\hline Langmuir [33] & $\begin{array}{l}\frac{C_{e}}{q_{e}} \\
=\frac{C_{e}}{q_{m}}+\frac{1}{K_{L} q_{m}}\end{array}$ & (5) & $C_{e} / q_{e}$ vs $C_{e}$ \\
\hline Temkin [58] & $\begin{array}{l}q_{e} \\
=\frac{R T}{b_{t}} \ln k_{T} \\
+\frac{R T}{b_{t}} \ln C_{e}\end{array}$ & (6) & $q_{e} v s \ln C_{e}$ \\
\hline $\begin{array}{l}\text { Dubinin } \\
\text { Radushkevich (D - } \\
\text { R [59] }\end{array}$ & $\begin{array}{l}\ln q_{e} \\
=\ln q_{m}-\beta \varepsilon^{2} \\
\quad \varepsilon \\
\quad=R T \ln (1 \\
\left.\quad+1 / C_{e}\right) \\
\quad E_{a}=1 / \sqrt{2} \beta\end{array}$ & $\begin{array}{l}(7) \\
(8) \\
(9)\end{array}$ & $\ln q_{e} v s \ln C_{e}$ \\
\hline $\begin{array}{l}\text { Sips (Langmuir - } \\
\text { Freundlich) [35] }\end{array}$ & $\begin{array}{l}\ln \left(\frac{q_{e}}{q_{m}-q_{e}}\right) \\
=n \ln C_{e} \\
+\ln K_{s}^{n}\end{array}$ & (10) & $\ln \left(\frac{q_{e}}{q_{m}-q_{e}}\right) v s \ln K_{s}^{n}$ \\
\hline $\begin{array}{l}\text { Redlich-Peterson } \\
\text { [35] }\end{array}$ & $\begin{array}{l}\ln \left(K_{R} \frac{C_{e}}{q_{e}}-1\right) \\
=n \ln C_{e}+\ln b\end{array}$ & (11) & $\ln \left(K_{R} \frac{C_{e}}{q_{e}}-1\right) v s \ln C_{e}$ \\
\hline
\end{tabular}

Where the $\mathrm{q}_{\mathrm{e}}$ is the sorption capacity at equilibrium $(\mathrm{mg} / \mathrm{g}) ; \mathrm{q}_{\mathrm{m}}$ is the maximum sorption capacity $(\mathrm{mg} / \mathrm{g}) ; \mathrm{C}_{\mathrm{e}}$ $(\mathrm{mg} / \mathrm{L})$ is the equilibrium concentration of biosorbent; $\mathrm{K}_{\mathrm{L}}$ is the Langmuir constant $(\mathrm{L} / \mathrm{mg}) ; \mathrm{K}_{\mathrm{F}}$ is the Freundlich constants of the adsorption capacity and exponent $\mathrm{n}$ is the adsorption intensity which varies with heterogeneity of the material. $\mathrm{K}_{\mathrm{s}}$ is the affinity constant for adsorption in Sips model $(\mathrm{L} / \mathrm{g}) ; \mathrm{K}_{\mathrm{R}}$ $(\mathrm{L} / \mathrm{g})$ and $\mathrm{b}(\mathrm{L} / \mathrm{mg})$ are the Redlich-Peterson constants of adsorption. $\mathrm{K}_{\mathrm{T}}$ is the Temkin's equilibrium binding constant corresponding to the maximum binding energy $(\mathrm{L} / \mathrm{mg}) ; b_{t}$ is the Temkin isotherm constant $\left(\mathrm{Jmol}^{-1}\right)$; $\mathrm{R}$ is the gas constant $\left(8.314 \mathrm{JK}^{-1} \mathrm{~mol}^{-1}\right)$; $\mathrm{T}$ is the temperature in Kelvin; $\boldsymbol{\varepsilon}$ is the Polanyi potential for adsorption; $E_{a}$ is the change in free energy when $1 \mathrm{~mol}$ of ion is transported to the surface of the solid in solution. It is calculated from $\beta$, which is the D-R constant. If the magnitude of $\mathrm{E}_{\mathrm{a}}$ is between 8 and $16 \mathrm{~kJ} / \mathrm{mol}$, then chemisorption process takes place and if the values of $E_{a}$ is less than $8 \mathrm{~kJ} / \mathrm{mol}$, then physisorption process occurs

The Langmuir isotherm model assumes that there is finite number of active sites distributed homogeneously over the surface of the adsorbent. The affinity of these active sites for adsorption is same towards a mono molecular layer and the interaction between adsorbed molecules is nil [37]. The biosorbents that undergo the respective isotherm models are summarized in table IV.

TABLE IV. ISOTHERM PARAMETERS OF THE VARIOUS BIOSORBENTS

\begin{tabular}{|c|c|c|c|}
\hline Biosorbent & $\begin{array}{c}\text { Isotherm } \\
\text { Model }\end{array}$ & Remarks & Ref. \\
\hline Peanut shells & Langmuir & $\mathrm{q}_{\mathrm{m}}=39 \mathrm{mg} \mathrm{g}^{-1}$ & {$[15]$} \\
\hline Rice Husk Ash & Langmuir & $\mathrm{q}_{\mathrm{m}}=0.0561 \mathrm{mmol} / \mathrm{g}$ & [24] \\
\hline \multirow[t]{2}{*}{ P. americana } & Freudlich & $\begin{array}{l}\mathrm{K}_{\mathrm{f}}=0.6006\left(\mathrm{mg} \mathrm{g}^{-1}\right)(\mathrm{L} \\
\left.\mathrm{mg}^{-1}\right)^{1 / \mathrm{n}} \\
1 / \mathrm{n}=0.6742\end{array}$ & \multirow{4}{*}{ [28] } \\
\hline & Langmuir & $\mathrm{q}_{\mathrm{m}}=10.83 \mathrm{mg} \mathrm{g}^{-1}$ & \\
\hline \multirow[t]{2}{*}{$\begin{array}{l}\mathrm{HNO}_{3} \text { modified } P . \\
\text { americana }\end{array}$} & Freudlich & $\begin{array}{l}\mathrm{K}_{\mathrm{f}}=1.244\left(\mathrm{mg} \mathrm{g}^{-1}\right)\left(\mathrm{L} \mathrm{mg}^{-}\right. \\
\left.{ }^{1}\right)^{1 / \mathrm{n}} \\
1 / \mathrm{n}=0.5906\end{array}$ & \\
\hline & Langmuir & $\mathrm{q}_{\mathrm{m}}=12.66 \mathrm{mg} \mathrm{g}-1$ & \\
\hline \multirow[t]{3}{*}{ Rapeseed biomass } & Langmuir & $\mathrm{q}_{\mathrm{m}}=18.35 \mathrm{mg} / \mathrm{L}$ at $4^{\circ} \mathrm{C}$ & \multirow{3}{*}{ [33] } \\
\hline & Langmuir & $\mathrm{q}_{\mathrm{m}}=21.29 \mathrm{mg} / \mathrm{L}$ at $20^{\circ} \mathrm{C}$ & \\
\hline & Langmuir & $\mathrm{q}_{\mathrm{m}}=22.7 \mathrm{mg} / \mathrm{L}$ at $25^{\circ} \mathrm{C}$ & \\
\hline \multirow{2}{*}{$\begin{array}{l}\text { Nanostructured cedar } \\
\text { leaf }\end{array}$} & Langmuir & $\mathrm{q}_{\mathrm{m}}=7.23 \mathrm{mg} \mathrm{g}^{-1}$ & \multirow{2}{*}{ [35] } \\
\hline & Sips & $\mathrm{q}_{\mathrm{m}}=8.045 \mathrm{mg} \mathrm{g}^{-1}$ & \\
\hline Corn husk & Langmuir & $\mathrm{q}_{\mathrm{m}}=3.034 \mathrm{mg} \mathrm{g}^{-1}$ & [37] \\
\hline $\begin{array}{l}\text { Cyclosorus } \\
\text { interruptus }\end{array}$ & Langmuir & $\mathrm{q}_{\mathrm{m}}=46.25 \mathrm{mg} \mathrm{g}^{-1}$ & [38] \\
\hline Raw maize stover & Langmuir & $\mathrm{q}_{\mathrm{m}}=19.65 \mathrm{mg} \mathrm{g}^{-1}$ & \multirow{2}{*}[40]{} \\
\hline Treated maize stover & Langmuir & $\mathrm{q}_{\mathrm{m}}=27.1 \mathrm{mg} \mathrm{g}^{-1}$ & \\
\hline Cashew nut & Langmuir & $\mathrm{q}_{\mathrm{m}}=11.23 \mathrm{mg} \mathrm{g}^{-1}$ & [41] \\
\hline Olive stone & Langmuir & $\mathrm{q}_{\mathrm{m}}=6.33 \mathrm{mg} \mathrm{g}^{-1}$ & \multirow{8}{*}{ [44] } \\
\hline $\begin{array}{l}\mathrm{HNO}_{3} \text { modified Olive } \\
\text { Stone }\end{array}$ & Langmuir & $\mathrm{q}_{\mathrm{m}}=49.13 \mathrm{mg} \mathrm{g}^{-1}$ & \\
\hline $\begin{array}{ll}\mathrm{H}_{2} \mathrm{SO}_{4} & \text { modified } \\
\text { Olive Stone } & \end{array}$ & Langmuir & $\mathrm{q}_{\mathrm{m}}=14.83 \mathrm{mg} \mathrm{g}^{-1}$ & \\
\hline $\begin{array}{l}\mathrm{NaOH} \text { modified Olive } \\
\text { Stone }\end{array}$ & Langmuir & $\mathrm{q}_{\mathrm{m}}=38.93 \mathrm{mg} \mathrm{g}^{-1}$ & \\
\hline Olive Tree Pruning & Langmuir & $\mathrm{q}_{\mathrm{m}}=26.72 \mathrm{mg} \mathrm{g}^{-1}$ & \\
\hline $\begin{array}{l}\mathrm{HNO}_{3} \text { modified Olive } \\
\text { Tree Pruning }\end{array}$ & Langmuir & $\mathrm{q}_{\mathrm{m}}=86.4 \mathrm{mg} \mathrm{g}^{-1}$ & \\
\hline $\begin{array}{l}\mathrm{H}_{2} \mathrm{SO}_{4} \quad \text { modified } \\
\text { Olive Tree Pruning }\end{array}$ & Langmuir & $\mathrm{q}_{\mathrm{m}}=72.78 \mathrm{mg} \mathrm{g}^{-1}$ & \\
\hline $\begin{array}{l}\mathrm{NaOH} \text { modified Olive } \\
\text { Tree Pruning }\end{array}$ & Langmuir & $\mathrm{q}_{\mathrm{m}}=123.8 \mathrm{mg} \mathrm{g}^{-1}$ & \\
\hline \multirow[t]{3}{*}{ S. obliquus } & Langmuir & $\mathrm{q}_{\mathrm{m}}=112 \mathrm{mg} \mathrm{g}^{-1}$ & \multirow{6}{*}{ [45] } \\
\hline & Freudlich & $\begin{array}{l}\mathrm{K}_{\mathrm{f}}=22.35\left(\mathrm{mg} \mathrm{g}^{-1}\right)\left(\mathrm{L} \mathrm{mg}^{-}\right. \\
\left.{ }^{1}\right)^{1 / \mathrm{n}} \\
1 / \mathrm{n}=0.768\end{array}$ & \\
\hline & $\mathrm{D}-\mathrm{R}$ & $\begin{array}{l}\beta=-0.64 \times 10^{-8} \mathrm{~mol}^{2} \mathrm{~J}^{-2} \\
\mathrm{q}_{\mathrm{m}}=31.6 \mathrm{mmol} / \mathrm{g} \\
\mathrm{E}_{\mathrm{a}}=8.84 \mathrm{~kJ} / \mathrm{mol}\end{array}$ & \\
\hline \multirow[t]{3}{*}{ Modified S. obliquus } & Langmuir & $\mathrm{q}_{\mathrm{m}}=207.2 \mathrm{mg} \mathrm{g}^{-1}$ & \\
\hline & Freudlich & $\begin{array}{l}\mathrm{K}_{\mathrm{f}}=78.10\left(\mathrm{mg} \mathrm{g}^{-1}\right)\left(\mathrm{L} \mathrm{mg}^{-}\right. \\
\left.{ }^{1}\right)^{1 / \mathrm{n}} \\
1 / \mathrm{n}=0.6019\end{array}$ & \\
\hline & $\mathrm{D}-\mathrm{R}$ & $\begin{array}{l}\beta=-0.46 \times 10^{-8} \mathrm{~mol}^{2} \mathrm{~J}^{-2} \\
\mathrm{q}_{\mathrm{m}}=24.9 \mathrm{mmol} / \mathrm{g} \\
\mathrm{E}_{\mathrm{a}}=10.44 \mathrm{~kJ} / \mathrm{mol}\end{array}$ & \\
\hline Lentil husk & Langmuir & $\mathrm{q}_{\mathrm{m}}=81.43 \mathrm{mg} \mathrm{g}^{-1}$ & [46] \\
\hline
\end{tabular}




\begin{tabular}{|c|c|c|c|}
\hline \multirow[t]{4}{*}{ Anabaena sphaerica } & Langmuir & $\mathrm{q}_{\mathrm{m}}=121.95 \mathrm{mg} \mathrm{g}^{-1}$ & \multirow{4}{*}{ [47] } \\
\hline & Freundlich & $\mathrm{K}_{\mathrm{f}}=28.28\left(\mathrm{mg} \mathrm{g}^{-1}\right)\left(\mathrm{L} \mathrm{mg}^{-}\right.$ & \\
\hline & & $1 / \mathrm{n}=0.2631$ & \\
\hline & $\mathrm{D}-\mathrm{R}$ & $\begin{array}{l}\beta=-0.2433 \times 10^{-8} \mathrm{~mol}^{2} \mathrm{~J}^{-2} \\
q_{\mathrm{m}}=1.104 \mathrm{mmol} \mathrm{g} \\
E_{a}=14.3 \mathrm{~kJ} / \mathrm{mol}\end{array}$ & \\
\hline \multirow{3}{*}{$\begin{array}{l}\text { Salvinia natans (at } \\
\text { temperature }=15^{\circ} \mathrm{C} \text { ) }\end{array}$} & Langmuir & $\mathrm{q}_{\mathrm{m}}=0.614 \mathrm{mmol} \mathrm{g}^{-1}$ & \multirow{6}{*}[48]{} \\
\hline & Freundlich & $\begin{array}{l}\mathrm{K}_{\mathrm{f}}=0.439 \mathrm{mmol} \mathrm{g}^{-1} \\
\mathrm{n}=2.964\end{array}$ & \\
\hline & $\mathrm{D}-\mathrm{R}$ & $\begin{array}{l}\beta=-0.022 \times 10^{-5} \mathrm{~mol}^{2} \mathrm{~J}^{-2} \\
q_{\mathrm{m}}=0.508 \mathrm{mmol} / \mathrm{g} \\
\mathrm{E}_{\mathrm{a}}=4.814 \mathrm{~kJ} / \mathrm{mol}\end{array}$ & \\
\hline \multirow{3}{*}{$\begin{array}{l}\text { Salvinia natans (at } \\
\text { temperature }=30^{\circ} \mathrm{C} \text { ) }\end{array}$} & Langmuir & $\mathrm{q}_{\mathrm{m}}=0.295 \mathrm{mmol} \mathrm{g}^{-1}$ & \\
\hline & Freundlich & $\begin{array}{l}\mathrm{K}_{\mathrm{f}}=0.245 \mathrm{mmol} \mathrm{g}^{-1} \\
\mathrm{n}=4.204\end{array}$ & \\
\hline & $\mathrm{D}-\mathrm{R}$ & $\begin{array}{l}\beta=-0.015 \times 10^{-5} \mathrm{~mol}^{2} \mathrm{~J}^{-2} \\
\mathrm{q}_{\mathrm{m}}=0.301 \mathrm{mmol} / \mathrm{g} \\
\mathrm{E}_{\mathrm{a}}=5.814 \mathrm{~kJ} / \mathrm{mol}\end{array}$ & \\
\hline \multirow{5}{*}{$\begin{array}{l}\text { Mimusops } \\
\text { leaves }\end{array}$} & Langmuir & $\mathrm{q}_{\mathrm{m}}=15.408 \mathrm{mg} \mathrm{g}^{-1}$ & \multirow{5}{*}{ [49] } \\
\hline & Freundlich & $\begin{array}{l}\mathrm{K}_{\mathrm{f}}=1.392 \mathrm{mg} \mathrm{g}^{-1}\left(\mathrm{mg} \mathrm{g}^{-1}\right) \\
\left(\mathrm{L} \mathrm{mg}^{-1}\right)^{1 / \mathrm{n}} \\
1 / \mathrm{n}=\end{array}$ & \\
\hline & $\begin{array}{l}\text { Redlich- } \\
\text { Peterson }\end{array}$ & $\begin{array}{l}\mathrm{A}=5.805 \mathrm{~L} / \mathrm{g} \\
\mathrm{B}=3.261 \mathrm{~L} / \mathrm{mg}\end{array}$ & \\
\hline & Temkin & $\begin{array}{l}\mathrm{A}_{\mathrm{T}}=2.03 \mathrm{~L} / \mathrm{mg} \\
\mathrm{b}_{\mathrm{T}}=865.71\end{array}$ & \\
\hline & $\mathrm{D}-\mathrm{R}$ & $\begin{array}{l}\beta=0.0099 \mathrm{~mol}^{2} \mathrm{~kJ}^{-2} \\
\mathrm{q}_{\mathrm{m}}=29.18 \mathrm{mg} / \mathrm{g} \\
\mathrm{E}_{\mathrm{a}}=7.106 \mathrm{~kJ} / \mathrm{mol}\end{array}$ & \\
\hline $\begin{array}{l}\text { Jatropha curcas } L . \\
\text { seed husk ash }\end{array}$ & Langmuir & $\mathrm{q}_{\mathrm{m}}=263.1 \mathrm{mg} \mathrm{g}-1$ & {$[57]$} \\
\hline
\end{tabular}

\section{THERMODYNAMICS OF ADSORPTION}

In order to assert the thermodynamic nature of lead biosorption, several basic parameters including change in free energy $\left(\Delta \mathrm{G}^{0}\right)$, enthalpy $\left(\Delta \mathrm{H}^{0}\right)$, entrophy $\left(\Delta \mathrm{S}^{0}\right)$ were found by researchers by the following formula.

$$
\begin{aligned}
& \Delta \mathrm{G}^{0}=-\mathrm{RT} \ln \mathrm{K} \\
& \ln \mathrm{K}=\frac{\Delta \mathrm{S}^{0}}{\mathrm{R}}-\frac{\Delta \mathrm{H}^{0}}{\mathrm{RT}}
\end{aligned}
$$

Where $\mathrm{T}$ is the temperature (Kelvin, $\mathrm{K}$ ); $\mathrm{R}$ is the ideal gas constant $\left(8.314 \mathrm{Jmol}^{-1} \mathrm{~K}^{-1}\right)$ and $\mathrm{K}$ is the thermodynamic equilibrium constant. According to the equation 12 and 13, $\Delta \mathrm{H}^{0}$ and $\Delta \mathrm{S}^{0}$ can be calculated from the slope and intercept of the plot of $\ln (\mathrm{K})$ versus $1 / \mathrm{T}$ respectively. The thermodynamic equilibrium constant is the ratio of the amount of adsorbate ( $\mathrm{mg}$ ) adsorbed per litre to the equilibrium concentration of the adsorbate in $\mathrm{mg} / \mathrm{L}$.

Negative $\Delta G^{0}$ indicates thermodynamic feasibility and spontaneity of the biosorption process. The magnitude of $\Delta \mathrm{H}^{0}$ indicates whether the biosorption is physical or chemical. The heat of adsorption for a physical reaction is around $2.1-20.9$ $\mathrm{kJmol}^{-1}$ and the energy of activation for chemical reaction (Ea) is in the range of $20.9-418.4 \mathrm{kJmol}^{-1}$ [37]. $\Delta \mathrm{S}^{0}$ indicates the randomness during biosorption.

C. interruptus [38] could biosorb lead chemically while peanut shells [15] and M. elengi leaves [49] manifested physical biosorption. Rice husk ash [24] and P. americana [28] were found to exhibit both physico-sorption and chemisorption process, exothermally and spontaneously, while the biosorption of lead by maize stover [40], $S$. melongena leaf powder [42], corn husk [37] and S. ilicifolium [51], was found to be spontaneous and endothermic.

\section{CONCLUSION}

The removal of lead by various biosorbents has been reviewed. The major demerits of conventional heavy metal removal techniques are high cost, low removal efficiency, increased chemical and biological effluent sludge, additional chemical requirements for regeneration of adsorption medium and difficulty in biosorbent recovery. Thus by using biosorbents, the problems caused by the conventional treatments can be minimized and the process can be performed in an environmental friendly way.

\section{REFERENCES}

[1] Agency for Toxic Substances and Disease Registry (ATSDR), "Toxicological profile for Lead", Atlanta, GA: U.S. Department of Health and Human Services, Public Health Service, 2007.

[2] E. K. Silbergeld, J. Sauk, M. Somerman, A. Todd, F. McNeill, B. Fowler, et al., "Lead in bone: storage site, exposure source, and target organ", Neurotoxicology, vol. 14 (2-3), pp. 225-36, Summer-Fall, 1993.

[3] P. S. L. Barry, and D. B. Mossman, "Lead concentrations in human tissues", Brit. J. industr. Med., vol. 27 (4), pp. 339351, Oct. 1970.

[4] C. M. A. Ademorati, "Pollution by Heavy metals," Environmental Chemistry and Toxicology, Foludex press Ibadan, pp. 171-172, 1996.

[5] A. Navas-Acien, E. Guallar, K. E. Silbergeld, J. S. Rothenberg, "Lead exposure and cardiovascular disease - a systematic review", Environ Health Perspect, vol. 115, pp. 472-482, 2007.

[6] K. Schumann, "The toxicological estimation of the heavy metal content $(\mathrm{Cd}, \mathrm{Hg}, \mathrm{Pb})$ in food for infants and small children,” Z. Ernahrungswiss, vol. 29, pp. 54-73, 1990.

[7] Theodore I. Lidsky, Jay S. Schneider, "Lead neurotoxicity in children: basic mechanisms and clinical correlates", Brain, $\begin{array}{lllll}\text { vol. } & 126 & \text { (1), } & \text { pp. } & 5-19,\end{array}$ http://dx.doi.org/10.1093/brain/awg014

[8] World Health Organization, "Ten chemicals of major public health concern", 2016, Retrieved from http://www.who.int/ipcs/assessment/public_health/chemical s_phc/en/ Assessed on 24th October 2016.

[9] European Union (Drinking Water) Regulations, Government Publications, Dublin, 2014. ISBN 978-1-4468-1806-0.

[10] U.S. Environmental Protection Agency (EPA), Washington, DC, "Lead and Copper Rule: A Revised Quick Reference Guide." Document no. EPA 816-F-08-018, June 1991 Retrieved https://nepis.epa.gov/Exe/ZyPDF.cgi?Dockey=60001N8P.tx t Assessed on 24th October 2016.

[11] World Health Organization, Lead in Drinking-water, Background document for development of WHO Guidelines for Drinking-water Quality, incorporating 1st and 2nd addenda, Vol.1, Recommendations. - 3rd ed, WHO, Geneva, Switzerland, 2011.

[12] L. Velásquez, and J. Dussan, "Biosorption and bioaccumulation of heavy metals on dead and living biomass of Bacillus sphaericus". J. Hazard. Mater. vol. 167 (1-3), pp. 713-6, 2009.

[13] N. Ahalya, T. V. Ramachandra, and R. D. Kanamadi, "Biosorption of Heavy Metals," Research Journal of Chemistry and Environment, vol. 7, pp. 71-79, 2003.

[14] Z. Aksu, Y. Sag, and T. Kutsal, "The biosorption of copper by $C$. vulgaris and Z. ramigera," Environ. Technol., vol. 13, pp. $579-586,1992$ 
[15] Ş. Taşar, F. Kaya, and A. Özer, "Biosorption of lead (II) ions from aqueous solution by peanut shells: Equilibrium, thermodynamic and kinetic studies", Journal of Environmental Chemical Engineering, vol. 2 (2), pp. 1018 1026 , June

2014. http://dx.doi.org/10.1016/j.jece.2014.03.015

[16] S. J. Rao, K. C. Rao, and G. Prabhakar, "Optimization of Biosorption Performance of Casuarina Leaf Powder for the Removal of Lead Using Central Composite Design", J. Environ. Anal. Toxicol., vol. 3, no. 2, pp. 166, 2013. http://dx.doi:10.4172/2161- 0525.1000166

[17] Romera, E., F. González, A. Ballester, M. L. Blázquez, and J. A. Munoz. "Comparative study of biosorption of heavy metals using different types of algae." Bioresource Technology, vol. 98, no. 17, pp. 3344-3353, 2007.

[18] Hasan, H. A., Abdullah, S. R. S., Kofli, N. T., and Yeoh, S. J., "Interaction of environmental factors on simultaneous biosorption of lead and manganese ions by locally isolated Bacillus cereus". Journal of Industrial and Engineering Chemistry, vol. 37, pp. 295-305, 2016.

[19] Lutts, S., Qin, P., and Han, R. M., "Salinity influences biosorption of heavy metals by the roots of the halophyte plant species Kosteletzkya pentacarpos", Ecological Engineering, vol. 95, pp. 682-689.

[20] Norton L., Baskaran K., and Mckenzie T., "Biosorption of zinc from aqueous solutions using biosolids", Adv. Environ. Res., vol. 8, pp. 629-35, 2004.

[21] Uslu G., Dursun A. Y., Ekiz H. I., Aksu Z., "The effect of $\mathrm{Cd}(\mathrm{II}), \mathrm{Pb}(\mathrm{II})$ and $\mathrm{Cu}(\mathrm{II})$ ions on the growth and bioaccumulation properties of Rhizopus arrhizus", Process Biochem., vol. 39, pp. 105-10, 2003.

[22] Bahadir T., Bakan G., Altas L., and Buyukgungor H., "The investigation of lead removal by biosorption: an application at storage battery industry wastewaters", Enzyme and Microbial Technology, vol. 41, no. 1, pp. 98-102, July 2007.

[23] Tunali, S., Cabuk, A. and Akar, T., "Removal of lead and copper ions from aqueous solutions by bacterial strain isolated from soil", Chemical Engineering Journal, vol. 115, no. 3, pp. 203-211, 2006.

[24] Vieira, M. G. A., A. F. de Almeida Neto, M. G. C. Da Silva, C. N. Carneiro, and A. A. Melo Filho, "Adsorption of lead and copper ions from aqueous effluents on rice husk ash in a dynamic system." Brazilian Journal of Chemical Engineering 31, no. 2, pp. 519-529, 2014.

[25] Berhe, S., Ayele, D., Tadesse, A. and Mulu, A., "Adsorption Efficiency of Coffee Husk for Removal of Lead (II) from Industrial Effluents: Equilibrium and Kinetic Study", International Journal of Scientific and Research Publications, vol. 5, no. 9, September 2015.

[26] Sheng P. X, Ting Y. P, Chen J. P, and Hong L. "Sorption of lead, copper, cadmium, zinc, and nickel by marine algal biomass: characterization of biosorptive capacity and investigation of mechanisms", J. Colloid Interface Sci., vol. 275, pp. 131-41, 2004

[27] Ma, X., Cui, W., Yang, L., Yang, Y., Chen, H. and Wang, K., "Efficient biosorption of lead (II) and cadmium (II) ions from aqueous solutions by functionalized cell with intracellular $\mathrm{CaCO}_{3}$ mineral scaffolds", Bioresource technology, vol. 185, pp.70-78, 2015. http://dx.doi.org/10.1016/j.biortech.2015.02.074

[28] Wang G., Zhang S., Yao P, Chen Y, Xu X, Li T, and Gong G., "Removal of $\mathrm{Pb}(\mathrm{II})$ from aqueous solutions by Phytolacca americana L. biomass as a low cost biosorbent", Arabian Journal of Chemistry, June 2015. http://dx.doi.org/10.1016/j.arabjc.2015.06.011
[29] Lodeiro P., Barriada J. L., Herrero R., Sastre D. E. and Vicente M. E., "The marine macroalga Cystoseira baccata as biosorbent for cadmium (II) and lead (II) removal: kinetic and equilibrium studies", Environ. Pollu., vol. 142, pp. 264 273, 2006.

[30] Low K. S., Lee C. K. and Liew S. C., "Sorption of cadmium and lead from aqueous solutions by spent grain", Process Biochem., vol. 36, pp. 59-64, 2000.

[31] McKay G. and Ho Y. S., "Pseudo-second-order model for sorption process", Process Biochem., vol. 34, pp. 451-465, 1999.

[32] I. Tsibranska, and E. Hristova, "Comparison of different kinetic models for adsorption of heavy metals onto activated carbon from apricot stones", Bulgarian Chemical Communications, vol. 43, no. 3, pp. 370 - 377, 2011.

[33] Morosanu, I., Teodosiu, C., Paduraru, C., Ibanescu, D. and Tofan, L., "Biosorption of lead ions from aqueous effluents by rapeseed biomass", New Biotechnology, 2016. http://dx.doi.org/10.1016/j.nbt.2016.08.002

[34] A. Maleki, E. Pajootan, and B. Hayati, "Ethyl acrylate grafted chitosan for heavy metal removal from wastewater: equilibrium, kinetic and thermodynamic studies", J. Taiwan Inst. Chem. Eng., vol. 51, pp. 127-134, 2015

[35] Hafshejani L. D., Nasab S. B., Gholami R. M., Moradzadeh M., Izadpanah Z., Hafshejani SB, et al., "Removal of zinc and lead from aqueous solution by nanostructured cedar leaf ash as biosorbent", Journal of Molecular Liquids, vol. 211, pp. 448-56, 2015 http://dx.doi.org/10.1016/j.molliq.2015.07.044.

[36] Langmuir, I., "The adsorption of gases on plane surfaces of glass, mica and platinum", J. American Chem. Soc., vol. 40, pp. 1361-1403, 1918.

[37] D. Sarkar, G. Paul and Ramachandra Murthy, T. T. S., "Studies on biochemical thermodynamics of lead biosorption from aqueous system using corn husk biomass as biosorbent agent", International Journal of Current Research, vol. 8, no. 09, pp. 37592-37598, 2016.

[38] Zhou K, Yang Z, Liu Y, and Kong X., "Kinetics and equilibrium studies on biosorption of $\mathrm{Pb}$ (II) from aqueous solution by a novel biosorbent: Cyclosorus interruptus", J. Environ. Chem. Eng. vol. 3, pp. 2219-28, 2015. doi:http://dx.doi.org/10.1016/j.jece.2015.08.002

[39] Moyo M., Guyo U., Mawenyiyo G., Zinyama N. P, and Nyamunda B. C., "Marula seed husk (Sclerocarya birrea) biomass as a low cost biosorbent for removal of $\mathrm{Pb}(\mathrm{II})$ and $\mathrm{Cu}$ (II) from aqueous solution", J. Ind Eng Chem, vol. 27, pp. 126-32,

2015 doi:http://dx.doi.org/10.1016/j.jiec.2014.12.026

[40] Guyo U., Mhonyera J., and Moyo M., "Pb(II) adsorption from aqueous solutions by raw and treated biomass of maize stover - a comparative study", Process Saf Environ Prot., vol. 93, pp. 192-200, 2015 http://dx.doi.org/10.1016/j.psep.2014.06.009

[41] Coelho G. F., Gonçalves Jr. A. C., Tarley C. R. T., Casarin J., Nacke H., Francziskowski M. A., "Removal of metal ions $\mathrm{Cd}$ (II), $\mathrm{Pb}$ (II), and $\mathrm{Cr}$ (III) from water by the cashew nut shell Anacardium occidentale L.", Ecol. Eng., vol. 73, pp. 514-25, http://dx.doi.org/10.1016/j.ecoleng.2014.09.103

[42] G. Yuvaraja, N. Krishnaiah, M.V. Subbaiah, and A Krishnaiah, "Biosorption of $\mathrm{Pb}$ (II) from Aqueous Solution by Solanum melongena Leaf Powder as a Low-Cost Biosorbent Prepared from Agricultural Waste", Colloids and Surfaces B: Biointerfaces, 2013. http://dx.doi.org/10.1016/j.colsurfb.2013.09.039 
[43] Martins, A. E., Pereira, M. S., Jorgetto, A. O., Martines, M. A., Silva, R. I., Saeki, M. J. and Castro, G. R., "The reactive surface of Castor leaf [Ricinus communis L.] powder as a green adsorbent for the removal of heavy metals from natural river water", Applied Surface Science, vol. 276, pp. 24-30, 2013

[44] Blázquez, G., Ronda, A., Martín-Lara, M.A., Pérez, A. and Calero, M., "Comparative study of isotherm parameters of lead biosorption by two wastes of olive-oil production", Water Science and Technology, vol. 72, no.5, pp.711-720, 2015.

[45] Abdel Ghafar, H.H., Abdel-Aty, A.M., Ammar, N.S. and Embaby, M.A., "Lead biosorption from aqueous solution by raw and chemically modified green fresh water algae Scenedesmus obliquus", Desalination and Water Treatment, vol. 52, no. 40-42, pp.7906-7914, 2014.

[46] Basu, M., Guha, A.K. and Ray, L., "Biosorptive removal of lead by lentil husk", Journal of Environmental Chemical Engineering, vol. 3, no. 2, pp.1088-1095, 2015. http://dx.doi.org/10.1016/j.jece.2015.04.024

[47] Abdel-Aty, A.M., Ammar, N.S., Ghafar, H.H.A. and Ali, R.K., "Biosorption of cadmium and lead from aqueous solution by fresh water alga Anabaena sphaerica biomass", Journal of advanced research, vol. 4, no. 4, pp.367-374, 2013. http://dx.doi.org/10.1016/j.jare.2012.07.004

[48] Lima L. K. S., Silva J. F. L., Da Silva M. G. C., Vieira M. G. A., "Lead biosorption by Salvinia natans biomass: equilibrium study", Chemical Engineering Transactions, vol. 38, pp. 97-102, 2014.

[49] Kalyani G., Prasanna Kumar Y. and King P., "Optimization of Lead Biosorption by an Ecofriendly Biosorbent Mimusops elengi Using Central Composite Design”, Research Journal of Pharmaceutical, Biological and Chemical Sciences, vol. 7, no. 3, pp. 1586-1603, 2016.

[50] Ibrahim, W. M., Hassan, A. F., and Azab, Y. A., "Biosorption of toxic heavy metals from aqueous solution by Ulva lactuca activated carbon", Egyptian Journal of Basic and Applied Sciences, vol. 3, no. 3, pp. 241-249, 2016. http//dx.doi.org/10.1016/j.ejbas.2016.07.005

[51] Tabaraki, R., Nateghi, A., and Ahmady-Asbchin, S., "Biosorption of lead (II) ions on Sargassum ilicifolium: Application of response surface methodology", International Biodeterioration \& Biodegradation, vol. 93, pp. 145-152, 2014.

[52] Siva Kiran R. R., Madhu G. M., Satyanarayana S. V., Kalpana P., Bindiya P., Subba Rangaiah G., "Equilibrium and kinetic studies of lead biosorption by three Spirulina (Arthrospira) species in open raceway ponds", J. Biochem Tech., vol. 6, no. 1, pp. 894-909, 2015.

[53] Masoumi, F., Khadivinia, E., Alidoust, L., Mansourinejad, Z., Shahryari, S., Safaei, M., et al., "Nickel and lead biosorption by Curtobacterium sp. FM01, an indigenous bacterium isolated from farmland soils of northeast Iran", Journal of Environmental Chemical Engineering, vol. 4, no. 1, pp.950-957, 2016.

[54] Gergana, K., Zdravka, V., Margarita, S., Yana, H., Iliev, I. and Velizar, G., "Biosorption of $\mathrm{Pb}$ (II) ions from aqueous solutions by waste biomass of Streptomyces fradiae pretreated with $\mathrm{NaOH}$ ", Biotechnology and Biotechnological Equipment (Bulgaria). vol. 29, no. 4, pp. 689-695, 2015.

[55] Amer, M.W., Ahmad, R.A. and Awwad, A.M., "Biosorption of $\mathrm{Cu}$ (II), Ni (II), $\mathrm{Zn}$ (II) and $\mathrm{Pb}$ (II) ions from aqueous solution by Sophora japonica pods powder", International Journal of Industrial Chemistry, 6(1), pp.67-75, 2015. DOI $10.1007 / \mathrm{s} 40090-014-0030-8$
[56] Alavi, S. A., Zilouei, H. and Asadinezhad, A., "Otostegia persica biomass as a new biosorbent for the removal of lead from aqueous solutions", Int. J. Environ. Sci. Technol., vol. 12, pp. 489 - 498, 2015. doi:10.1007/s13762-014-0705-x

[57] Shi, B., Zuo, W., Zhang, J., Tong, H. and Zhao, J., "Removal of Lead (II) Ions from Aqueous Solution Using Jatropha curcas L. Seed Husk Ash as a Biosorbent", Journal of environmental quality, vol. 45, no. 3, pp. 984-992, 2016.

[58] Aharoni A. and Ungarish M., "Kinetics of activated chemisorptions Part 2. Theoretical models", J Chem Soc Faraday Trans., vol. 73, pp. 456-464, 1977.

[59] Huston N. D. and Yang R. T., "Theoretical basis for the Dubinin-Radushkevich (D-R) adsorption isotherm equation", Adsorption, vol. 3, pp. 189-195, 1997. 